DOI: $10.3901 / J M E .2019 .18 .104$

\title{
高速动车组齿轮传动系统振动特性 ${ }^{*}$
}

\author{
孙 刚 任尊松 辛欣 魏雪 \\ (北京交通大学机械与电子控制工程学院＼cjkstart北京 100044)
}

\begin{abstract}
摘要: 齿轮传动系统是高速动车组传递动力的关键结构, 对车辆运行安全性和结构振动及其受载特性有重要影响, 研究高速 列车传动系统的动力学性能对提升高速动车组技术、提高车辆系统稳定性和安全性等具有重要意义。采用有限元法、自由度 缩减理论以及多体动力学理论, 建立了包含齿轮传动系统振动的国内某型高速动车组刚柔耦合系统动力学模型。模型中, 车 体和轮对处理为刚体, 构架和齿轮副处理为弹性体。在验证模型齿轮副啮合特性基础上, 研究了多种工况下传动系统的动力 学性能及其与车辆系统主要部件之间的动态相互作用, 获得了传动系统载荷及其对车辆系统主要部件振动的影响特性。结果 表明, 轨道激扰和车辆运行速度对传动系统振动有明显影响, 传动系统振动对构架、齿轮箱以及电动机振动等有一定的影响。 建立的高速车辆-传动系统耦合振动模型, 突破了传统轨道车辆动力学模型模式, 对深入研究高速动车组传动系统振动特性具 有重要作用。
\end{abstract}

关键词: 高速动车组系统动力学模型; 传动系统动力学模型; 齿轮啮合; 载荷; 振动 中图分类号: U270

\section{Dynamics of Gear Transmission System of High-speed Vehicle}

\section{SUN Gang REN Zunsong XIN Xin WEI Xue}

(College of Mechanical and Electronic Control, Beijing Jiaotong University, Beijing 100044)

\begin{abstract}
The gear transmission system of the railway vehicle is the key part to transmit power for the Electric Multiple Unit(EMU). Its dynamic vibration affect the operation safety and the dynamic characteristics of the vehicle. It is necessary to study the dynamic characteristics of transmission system of the high-speed EMU to obtain the forces and accelerations of the gears. Based on the parameters of a certain type of $\mathrm{CRH}$, a rigid-flexible coupling dynamics model of railway vehicle is established. A flexible transmission system dynamics model is setup and it is included in the vehicle system dynamics model. With numerical method, the dynamics of the transmission system is obtained. The simulation results show that the forces acted on the gears get larger when the operation speed of the vehicle increases. The vibration of the transmission system affect the vibrations of the bogie frame, motor and gearbox, but has hardly effects on the vibration of the carbody because of the secondary suspension system and the huge mass of the carbody. The new railway vehicle-gear transmission coupling dynamics model is useful to investigate the dynamic characteristics of the gear transmission system of the high-speed train.
\end{abstract}

Key words: high speed EMU dynamics model; transmission system dynamics model; gear meshing; forces; vibration

\section{0 前言}

相对于其他交通方式，轨道交通具有经济、环 保、安全、便捷及高效等优势，在现代交通运输中 占据重要地位。车辆诨引动力通过牵引电动机、联 轴器再经过齿轮副传递给轮对 ${ }^{[1]}$, 进而保障车辆高 速运行, 因此齿轮传动系统是高速列车传递动力的 关键结构。

车辆运行过程中, 由于齿轮啮合刚度的时变特

* 国家自然科学基金资助项目(11790281，51575036)。20180607 收到初稿, 20190102 收到修改稿
性和存在传递误差，使得传动系统齿轮间产生内部 动态激励, 轮轨激励对高速车辆传动系统振动产生 明显影响, 高速车辆结构振动存在相互传递和影响。 因此, 传动系统激励的输入源十分丰富, 导致高速 车辆传动系统振动十分复杂, 进而对车辆其他结构 有着不可忽视的影响。

目前, 常规高速车辆系统动力学研究已逐步完 善, 但在车辆关键结构研究方面, 大多数研究主要 将研究对象集中在车体、构架及轮对等部件上 ${ }^{[2-5]}$, 对于高速车辆系统中齿轮系统、电动机等重要部件 振动一直未有详细研究。CHEN 等 ${ }^{[6]}$ 建立了包括齿 轮传动系统某型机车垂向动力学模型, 研究了齿轮 
扭转振动与机车振动相互作用问题, 但这种振动不 包括车辆和齿轮系统横向相互作用。HU 等 ${ }^{[7]}$ 采用有 限元计算和线路试验方法, 研究了齿轮箱箱体疲劳 失效问题, 但没有涉及齿轮副振动。 TANG 等 ${ }^{[8]}$ 采 用有限元法, 探讨了 $\mathrm{CRH} 380 \mathrm{~A}$ 型动车组多状态下 齿轮动态啮合特性。AL-SHYYABA ${ }^{[9]}$ 采用多项谐波 平衡方法, 分析了列车多轴齿轮啮合非线性动力学 问题。WANG 等 ${ }^{[10]}$ 考虑齿轮系统的传动误差和时变 刚度等因素, 建立了机车齿轮系统三自由度模型, 研究了齿轮系统非线性振动特性。

传动系统对高速车辆动力学性能的影响复杂多 样, 但既有研究大多是通过分析其主要影响因素, 提取其激励并等效到其他结构中代表传动系统的作 用, 虽也有将传动系统作为独立部件进行分析 ${ }^{[6,11]}$, 但是没有将传动系统与整车系统结合起来开展分析 和研究, 或者没有考虑其横向振动问题。

借助车辆系统动力学理论、有限元方法以及机 械动力学理论等, 这里建立了包含弹性传动系统在 内的国内某型高速动车组车辆系统刚柔耦合动力学 模型, 拟深入研究齿轮传动系统部件的振动特性和 高速车辆系统动态行为, 获得传动系统对车辆系统 振动的影响特性, 进而全面掌握高速车辆传动系统 的动力性能。

\section{1 高速动车组及传动系统动力学模型}

建立车辆系统动力学模型, 实质上是确定车辆 系统部件受载特征并建立其运动方程。通过数值方 法求解这些运动方程, 可获得系统振动的时间历程。

\section{1 传动系统模型}

高速动车组传动系统的主要传动过程是齿轮箱 内部的齿轮啮合传动和联轴器传动 ${ }^{[12]}$, 合理的齿轮 箱连接定义、齿轮啮合关系以及联轴器传动描述, 对建立正确的高速车辆传动系统动力学模型至关重 要。为准确反映传动系统的振动特性, 这里采用具 有质量和转动惯量的结构代表其惯性特征, 利用运 动方程表述其运动关系。

关于齿轮箱的固定, 通过 SIMPACK 中铰接来 进行基准位置的定位, 与轮轴、小齿轮轴的轴承连 接和与构架的橡胶垫连接采用 SIMPACK 中力元(刚 度、阻尼、自由度)统一定义。根据连接特性定义参 数, 齿轮箱可绕车轴转动。这种处理方式忽略了轴 承转动摩擦力影响, 能够有效模拟车辆运行过程中 振动的传递效果。

大齿轮和小齿轮的啮合在 SIMPACK 中是采用 225 号力元建立模型, 该力元能够准确描述齿轮传
动模型中齿面修型、齿侧间隙以及啮合刚度的时变 特性等。针对国内某型高速动车组, 该力元需要的 齿轮副轮廓参数和三维几何特性参数如表 1 所示。

表 1 高速动车组齿轮副参数

\begin{tabular}{cc||cc}
\hline 参数 & 数值 & 参数 & 数值 \\
\hline 小齿轮齿数 & 29 & 大齿轮齿数 & 73 \\
小齿轮变位 $/ \mathrm{mm}$ & 0.225 & 大齿轮变位 $/ \mathrm{mm}$ & 0.024 \\
模数 $/ \mathrm{mm}$ & 7 & 法向压力角 $/\left(^{\circ}\right)$ & 26 \\
小齿轮齿宽 $/ \mathrm{mm}$ & 70 & 大齿轮齿宽 $/ \mathrm{mm}$ & 69 \\
螺旋角 $/\left({ }^{\circ}\right)$ & 20 & 齿侧间隙 $/ \mathrm{mm}$ & 0 \\
啮合刚度比 & 0.8 & 泊松 & 0.3 \\
弹性模量 $/(\mathrm{GN} / \mathrm{m})$ & 210 & 阻尼系数 $/(\mathrm{kN} \cdot \mathrm{s} / \mathrm{m})$ & 5 \\
\hline
\end{tabular}

联轴器作为电动机传递扭矩至齿轮的关键结构 连接电动机和齿轮, 并对电动机和齿轮箱之间振动 有传递和衰减作用。根据联轴器结构和功能, SIMPACK 中采用 43 号力元建立联轴器模型, 并设 置合理的扭转刚度以确保联轴器扭矩的传递功能。

图 1 为依据齿轮参数、齿轮箱参数以及联轴器参数 等建立的齿轮副传动系统动力学模型。

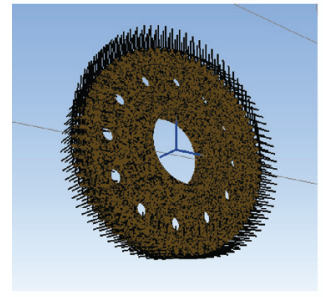

(a) 大齿轮弹性模型

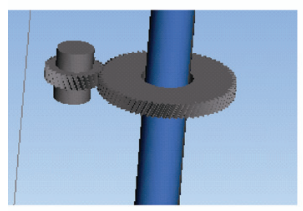

(c) 齿轮啮合传动

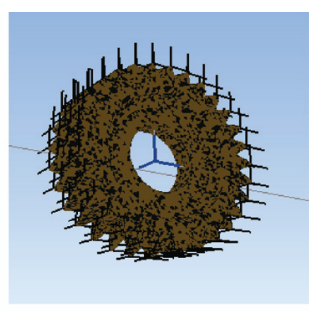

(b) 小齿轮弹性模型

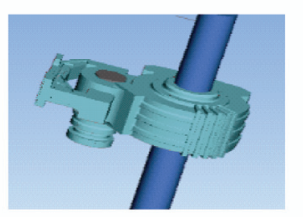

(d) 联轴节与齿轮箱
图 1 传动系统动力学模型

为获得准确的齿轮啮合振动特性, 这里借助有 限元法和自由度减缩理论等, 将传动系统中大小齿 轮均处理为弹性体, 具体的处理方法可参见相关文 献[3]。需要说明的是, 齿轮啮合传动时齿轮面为接 触面, 接触应力较大, 弹性变形也较大, 因此大小 齿轮缩减自由度的主节点主要设置在齿上。这里, 大齿轮选取 292 个主节点, 小齿轮选取 116 个主节 点。当齿轮间有相互作用力时, 齿轮上节点将产生 位移差, 用以表述齿轮的接触变形。因此, 啮合受 载状态下, 大小齿轮和轮齿均可以发生弹性变形和 振动。

\section{2 车辆系统动力学模型}

考虑车辆系统主要部件振动和悬挂系统的连接 
作用 ${ }^{[2]}$, 以国内某型高速动车组车辆为研究对象, 在 SIMPACK 中建立的该型车辆转向架模型如图 2 所示, 车辆系统动力学模型如图 3 所示。

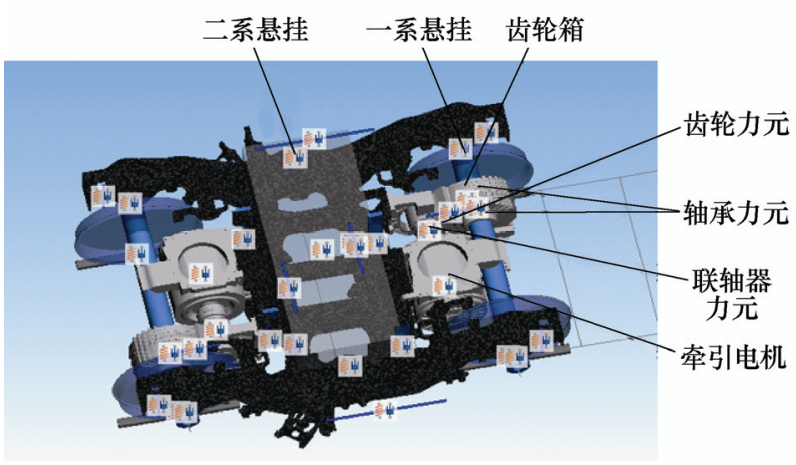

图 2 转向架动力学模型

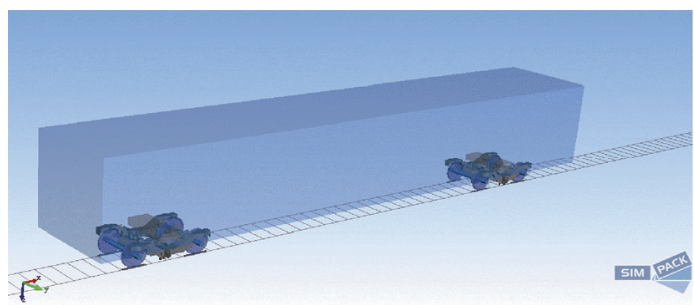

图 3 车辆系统动力学模型

模型中，车体和轮对处理为具有 6 自由度的刚 体, 构架处理为弹性体。构架弹性处理方式与齿轮 弹性处理方式一致。悬挂系统对这三大部件的约束 作用以弹簧-减振器单元描述, 即以载荷的方式作用 于相关连接部件。轮轨接触关系和减振器阻尼考虑 其非线性特性。轨道激扰为武广实测线路谱。需要 说明地是，模型中不考虑轨道系统振动。

建立包含弹性传动系统的刚柔耦合车辆系统动 力学模型后, 采用数值积分方法, 即可获得车辆系 统各主要部件在多种工况下的时间历程, 进而获得 其频率特性。此外, 为对比分析传动系统啮合振动 对车辆系统振动的影响, 本文计算了传动系统不啮 合振动工况下车辆系统的动态响应。

\section{2 齿轮副啮合振动特性}

图 4 给出了车辆运行速度为 $200 \mathrm{~km} / \mathrm{h}$ 时齿轮副 啮合刚度的时间历程。该组斜齿轮的平均啮合刚度 为 $1.48 \mathrm{GN} / \mathrm{m}$, 该值与实际齿轮啮合刚度吻合 ${ }^{[12]}$ 。 啮合刚度总体呈现周期时变特性, 符合静力学齿轮 啮合刚度的时变特性。受轨道激扰影响, 齿轮副啮 合刚度呈现局部无规则微小变化, 符合外界激励对 于齿轮啮合刚度的影响特性。计算过程中如果齿轮 啮合刚度突变到零, 表明该齿轮副没有啮合, 即大 齿轮与小齿轮脱离。需要说明的是, 这里的啮合刚
度是根据齿轮系统参数建立大小齿轮柔性动力学模 型后，通过约束关系系统自动确定。

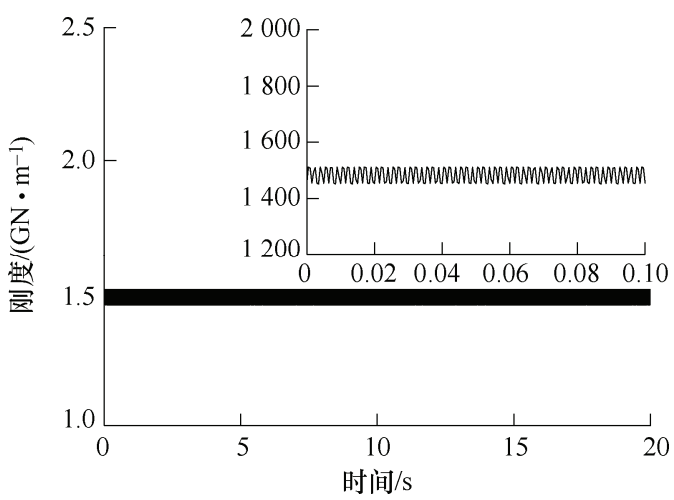

图 4 齿轮啮合刚度时间历程

图 5 给出了车辆速度为 $200 \mathrm{~km} / \mathrm{h}$ 时齿轮副啮合 齿数随时间的变化历程。由此可见，齿轮副的啮合 齿数呈现周期时变特性，表现为二齿或者三齿交替 啮合。或者说，正常状态下齿轮副啮合齿数的周期 变化特性能够得到充分体现。

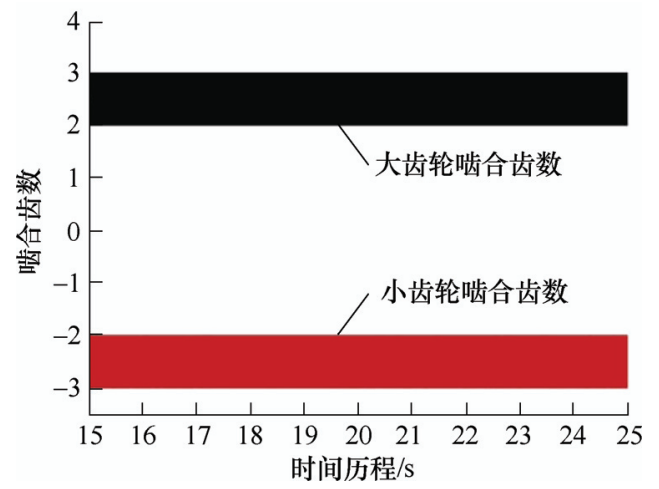

图 5 齿轮啮合齿数随时间的历程

由上述结果和分析可知，本文建立的齿轮副模 型反映了齿轮的啮合刚度特性和啮合特性 ${ }^{[13-14]}$, 具 有很好的合理性, 可以利用其开展进一步研究。

图 6 给出了齿轮系统在无外界激励下的齿轮啮 合力。由于无外界激励干扰，齿轮啮合传递误差小， 产生的动态啮合力幅值小、稳定性好。

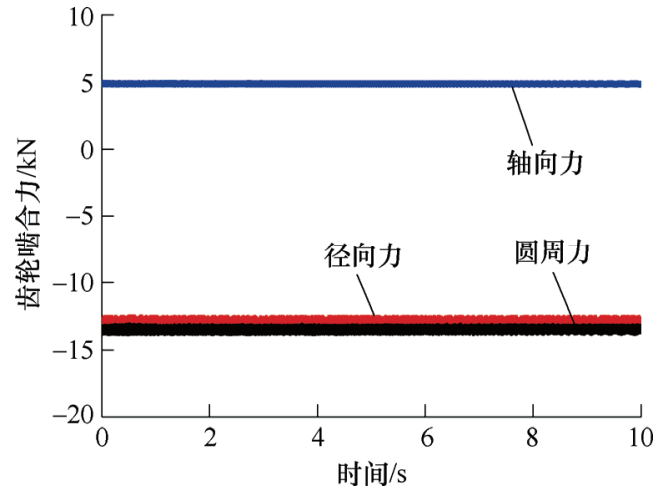

图 6 无轮轨激扰下齿轮啮合力时间历程 
图 7 给出了车辆前位转向架前齿轮传动系统 在速度分别为 $100 \mathrm{~km} / \mathrm{h} 、 200 \mathrm{~km} / \mathrm{h}$ 和 $300 \mathrm{~km} / \mathrm{h}$ 工 况下圆周力、径向力以及轴向力的时间历程及功 率谱密谱分布。对比图 6 可知, 轨道激扰作用下 齿轮系统的啮合力发生了明显波动, 且车辆速度 越高, 载荷波动幅值越大, 激振程度越明显。啮 合频率及其倍频, 在其功率谱密度图示中得到了 明显体现。相对于无激扰工况齿轮系统的圆周力、 径向力和轴向力, 在 $300 \mathrm{~km} / \mathrm{h}$ 速度且有武广线路 激扰作用下，这些载荷的最大值分别增大 1.88 、

0.71 和 1.90 倍左右。

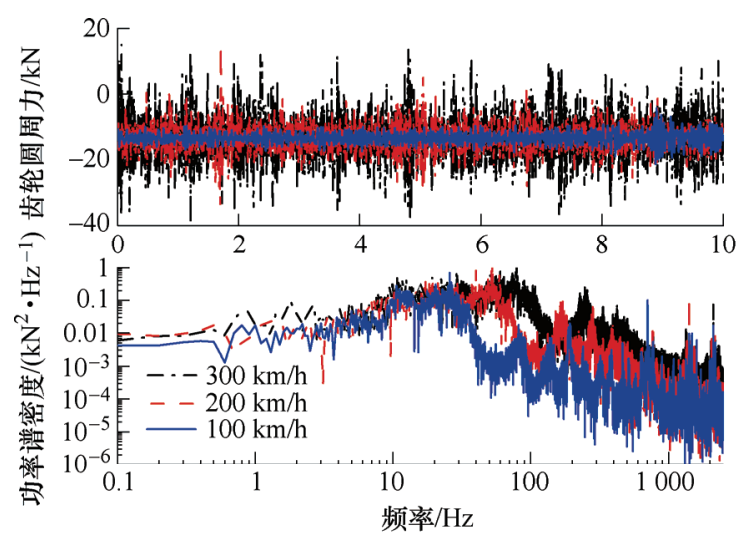

(a) 齿轮圆周力及功率谱密度

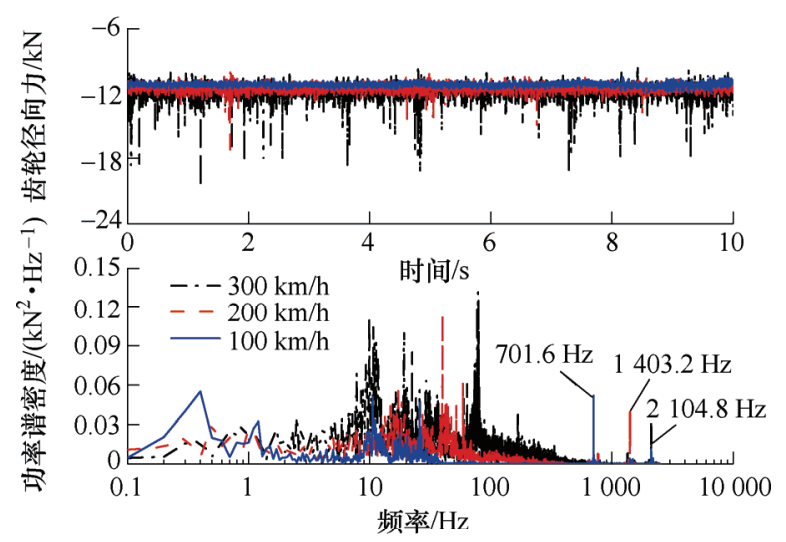

(b) 齿轮径向力及功率谱密度

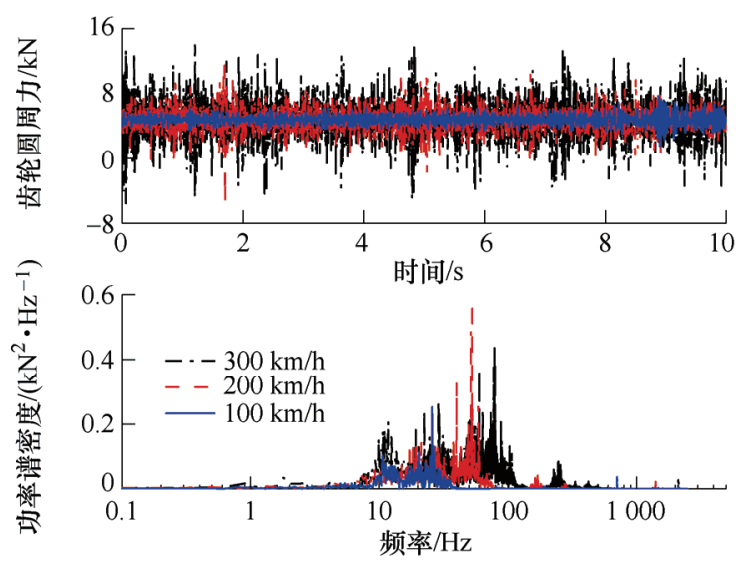

(c) 齿轮轴向力及功率谱密度

图 7 武广激扰下齿轮啮合力时间历程及功率谱密度
需要说明的是, 齿轮载荷在 $100 \mathrm{~km} / \mathrm{h} 、 200 \mathrm{~km} / \mathrm{h}$ 和 $300 \mathrm{~km} / \mathrm{h}$ 速度等级下分别在 $701.6 \mathrm{~Hz}, 1403.2$ $\mathrm{Hz}, 2104.8 \mathrm{~Hz}$ 频率处有比较高的波峰值, 这与齿 轮啮合频率密切相关。设 $V$ 为车辆运行速度、 $N$ 为 齿轮齿数、 $D$ 为车轮直径, 即有啮合频率 $f_{c}$

$$
f_{c}=\frac{V \cdot N}{\pi \cdot D}
$$

式中, 若取 $V=100 \mathrm{~km} / \mathrm{h}$ 、大齿轮齿数 $N=73$ 、直径 $D=0.92 \mathrm{~m}$, 可得到

$$
f_{c}=\frac{100 \times 73}{3.6 \times \pi \times 0.92}=701.6 \mathrm{~Hz}
$$

\section{3 传动系统对车辆动力学性能影响}

通过建立完整的传动系统模型, 可探究高速传 动结构系统对车辆各部件动力学的影响。需要说明 地是, 本部分对应的车辆运行速度为 $300 \mathrm{~km} / \mathrm{h}$, 且 有武广线路激扰。

\section{1 传动系统振动对车体振动影响}

图 8 给出了车辆高速直线运行时, 车体垂向加 速度在有无传动系统激励时的时域和频域特性。由 此可见, 传动系统激励对于车体垂向加速度影响非 常小。另外, 车体垂向振动频率主要分布在 $0 \sim 10 \mathrm{~Hz}$ 范围内, 一般不超过 $100 \mathrm{~Hz}$ 。

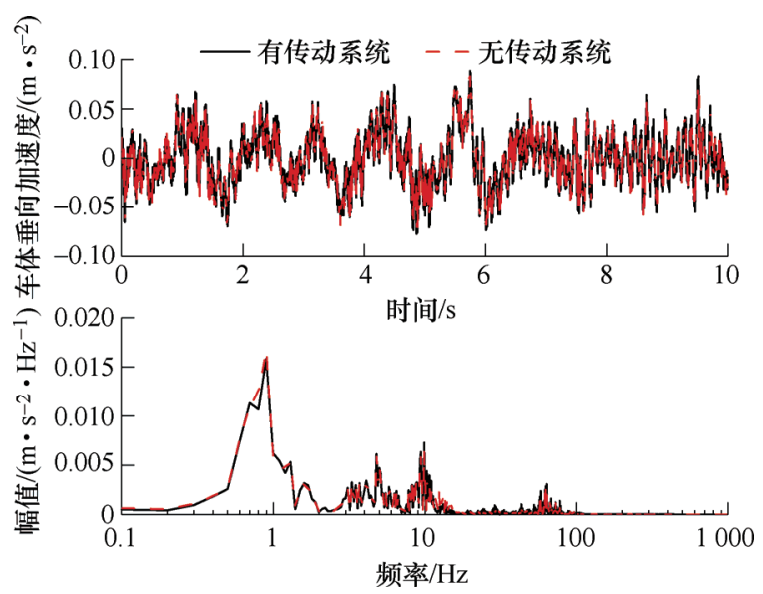

图 8 车体垂向加速度

图 9 给出了车辆高速直线运行时, 车体横向 加速度在传动系统激励作用下的时域和频域特 性。由此可见, 有齿轮传动振动时的车体横向加 速度幅值, 略大于无传动系统振动时的横向加速 度幅值。车体横向加速度的主要振动频率为 0 $100 \mathrm{~Hz}$, 比车体垂向加速度的主频范围更宽。相 对于无传动振动, 有传动振动时车体振动加速 度在 $10 \sim 100 \mathrm{~Hz}$ 范围内的振动能量有一定程度地 增加。 


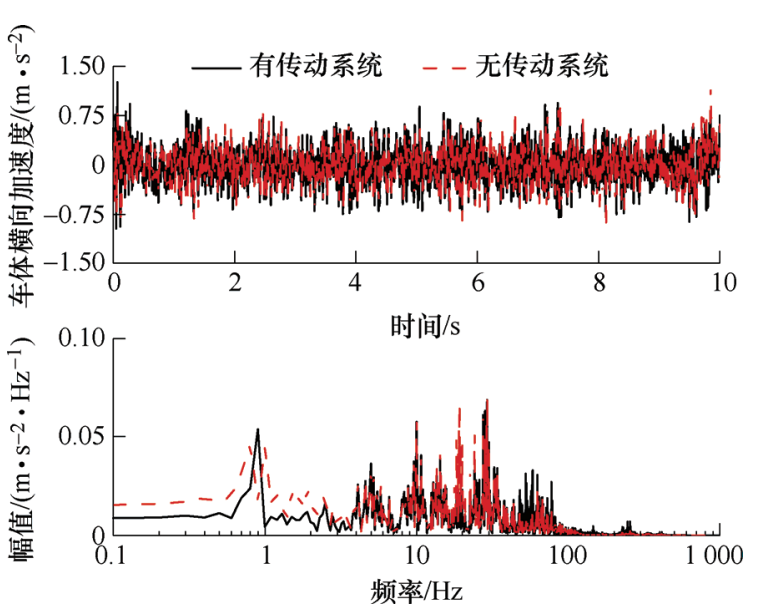

图 9 车体横向加速度

\section{2 传动振动对构架振动影响}

构架作为连接车体和轮对的重要部件, 其垂向 加速度受到多方面因素的影响, 这些因素也包括传 动系统振动。图 10、11 分别给出了车辆高速直线运 行时, 构架垂向和横向振动加速度的时间历程和频 域分布特性。由此可见, 在传动激励影响下, 构架 振动加速度幅值比无传动激励时的振动幅值大, 说 明传动系统振动增大了构架振动。

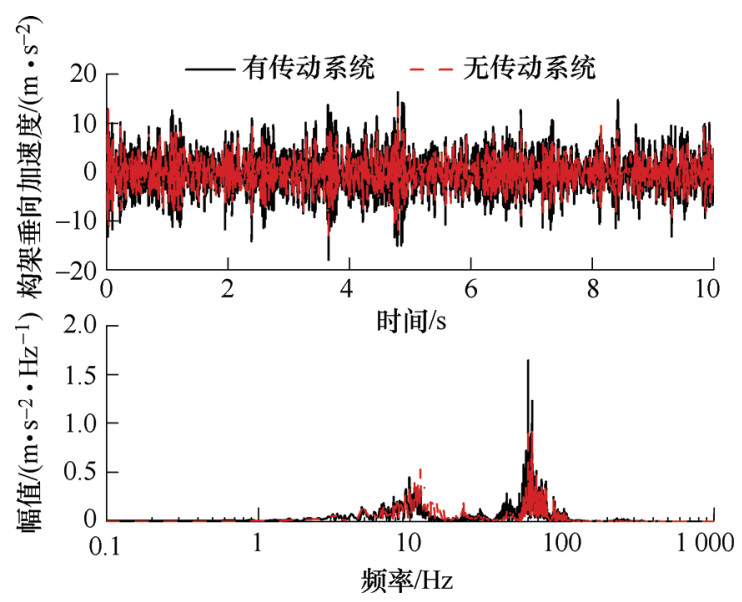

图 10 构架垂向加速度

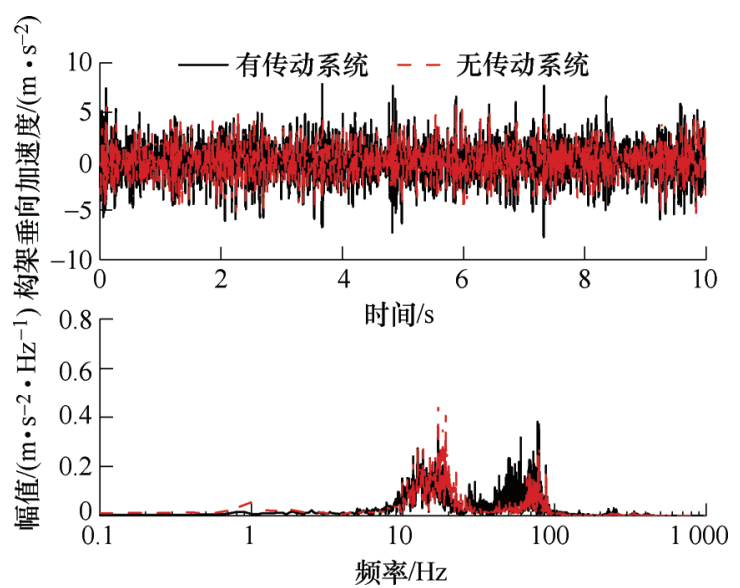

图 11 构架横向加速度

总体上, 传动系统振动对构架振动的影响, 大
于对车体振动的影响, 这与二系悬挂系统对传递至 车体的振动有明显的抑制和衰减作用、且车体质量 远大于构架质量密切相关。

\section{4 传动系统对齿轮箱振动影响}

高速列车齿轮箱箱体与多个部件连接, 这些连 接部件对其动力学性能均有影响。或者说, 齿轮箱 受到来自多个部件的载荷共同作用且这些载荷具有 时变性 ${ }^{[15-16]}$, 因此, 齿轮箱实际服役状态与常规静 力学研究方法对应的状态存在较大差异 ${ }^{[17-20]}$ 。有鉴 于此, 本文借助建立的包含传动系统的高速动车组 动力学模型, 探究传动系统激励对高速动车组齿轮 箱振动的影响。

图 12 给出了齿轮箱垂向振动加速度的时间历 程和频域分布特性。由此可见，传动系统啮合振动 对齿轮箱垂向振动有一定的影响, 但不明显。齿轮 箱垂向振动加速度频率主要分布在 $0 \sim 500 \mathrm{~Hz}$ 。在 $2100 \mathrm{~Hz}$ 左右的高频区段存在振动能量峰值, 这表 明齿轮啮合对齿轮箱高频振动有影响。

图 13 给出了齿轮箱横向振动加速度的时间历

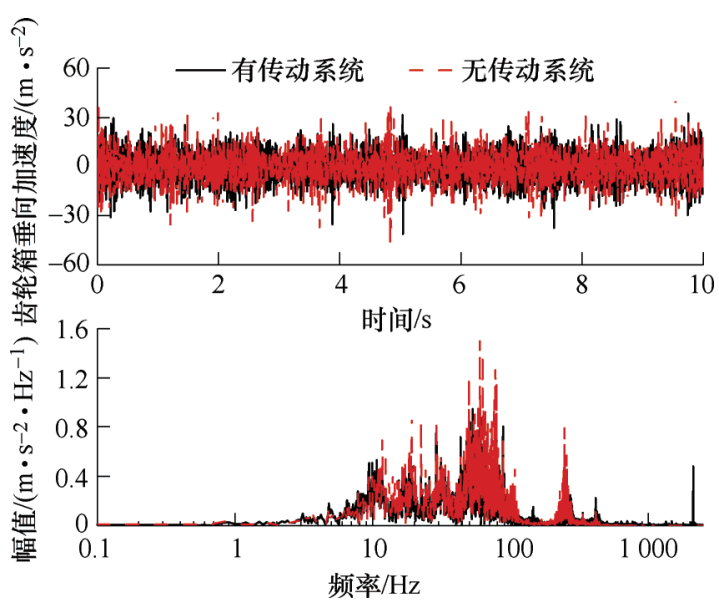

图 12 齿轮箱垂向加速度

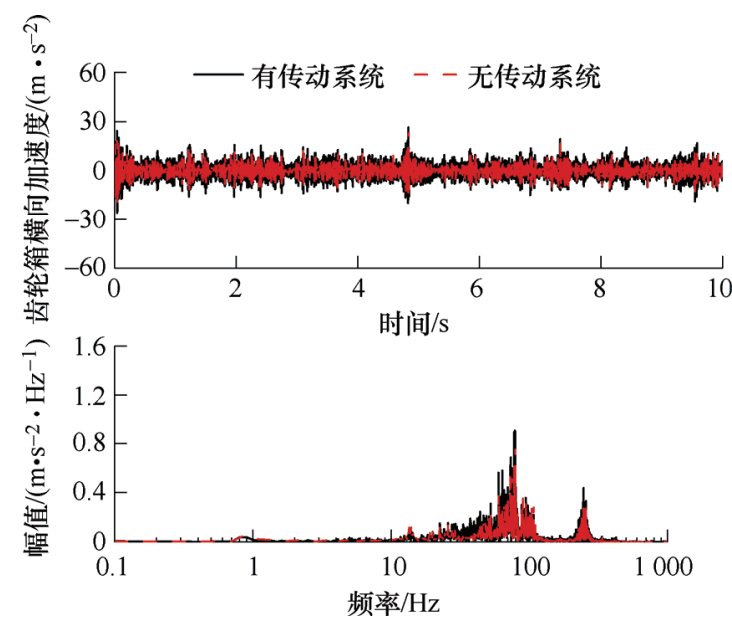

图 13 齿轮箱横向加速度 
程和频域分布特性。由此可见, 齿轮啮合振动对齿 轮箱体横向振动有较明显影响, 表现在齿轮箱箱体 振动加速度幅值增大、功率谱密度幅值增加等。与 垂向振动特性类似, 齿轮箱横向振动加速度主要分 布在 $0 \sim 500 \mathrm{~Hz}$ 范围内。

表 2 对比了两种工况下齿轮箱横向和垂向振动 加速度幅值。由前述可知, 齿轮啮合振动主要是增 大了齿轮箱高频振动能量, 使得齿轮箱的振动频率 范围更宽, 但对于齿轮箱的振动幅值影响较小。实 际上, 齿轮箱振动主要是受到轮对振动和构架振动 的影响明显, 而轮对和构架的振动源自轮轨激扰, 如车轮缺陷、轨道激扰以及车辆蛇行运动等。

表 2 齿轮箱加速度统计结果

\begin{tabular}{cccccccc}
\hline \multirow{2}{*}{ 工况 } & \multicolumn{3}{c}{ 垂向加速度 $/\left(\mathrm{m} / \mathrm{s}^{2}\right)$} & & \multicolumn{3}{c}{ 横向加速度 $/\left(\mathrm{m} / \mathrm{s}^{2}\right)$} \\
\cline { 2 - 3 } \cline { 7 - 8 } & 最大值 & 最小值 & 幅值 & & 最大值 & 最小值 & 幅值 \\
\hline 有传动 & 36.17 & -37.38 & 36.77 & & 26.52 & -25.75 & 26.13 \\
无传动 & 43.27 & -47.75 & 45.51 & & 25.99 & -23.45 & 24.72 \\
\hline
\end{tabular}

\section{5 传动系统对电动机振动影响}

电动机为高速动车组提供牵引动力, 其工作状 态影响动力输出的稳定性, 因此, 研究影响电动机 的振动因素是十分必要的。本文模型中, 电动机与 电动机吊架铰接联结, 因此电动机的载荷输入主要 有齿轮啮合激励通过联轴器传入电动机转子、构架 振动通过吊杆传递到电动机吊架等。

图 14、15 给出了两种工况下电动机垂向和横向 加速度的时间历程和频域分布特性。由此可见, 传 动系统主要增加了电动机在 $20 \sim 100 \mathrm{~Hz}$ 频域内的 振动幅值, 使得电动机振动在该频率范围内的振动 能量更高。超过 $100 \mathrm{~Hz}$ 后, 传动激励对于电动机高 频振动几乎无影响, 说明联轴器和电动机吊架有效 降低或拟制了齿轮啮合产生的高频振动对于电动机 的影响。

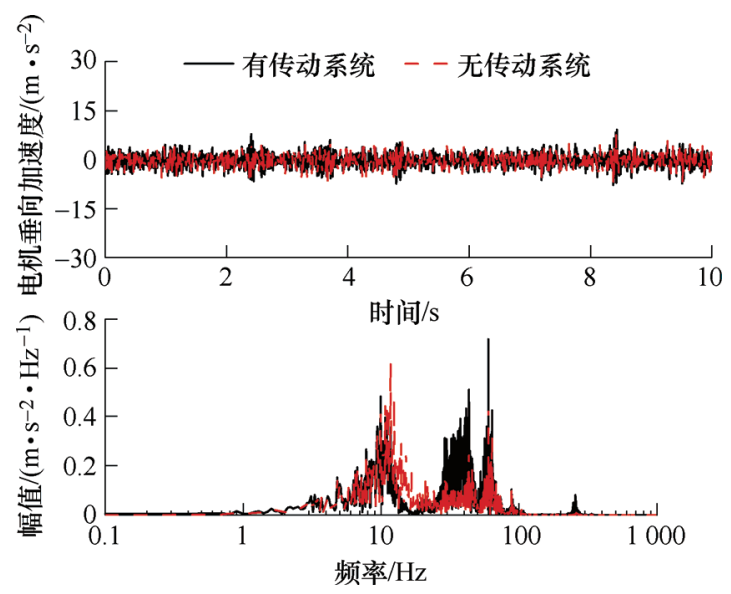

图 14 电动机垂向加速度
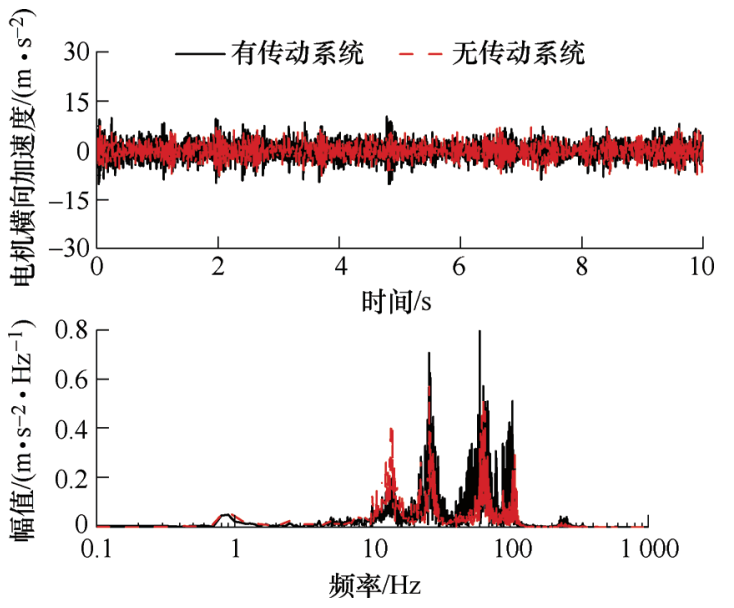

图 15 电动机横向加速度

表 3 给出了传动激励对电动机振动加速度幅值 影响情况。传动振动在一定程度上增大了电动机垂 向和横向加速度的幅值, 垂向加速度幅值增加 $5.67 \%$ 、横向加速度幅值增大 $25.67 \%$ 左右。

表 3 传动激励对电动机加速度影响

\begin{tabular}{ccccccccc}
\hline \multirow{2}{*}{ 工况 } & \multicolumn{3}{c}{ 垂向加速度 $/\left(\mathrm{m} / \mathrm{s}^{2}\right)$} & & \multicolumn{3}{c}{ 横向加速度 $/\left(\mathrm{m} / \mathrm{s}^{2}\right)$} \\
\cline { 2 - 4 } & 最大值 & 最小值 & 幅值 & & 最大值 & & 最小值 & 幅值 \\
\hline 有传动 & 9.40 & -7.45 & 8.42 & & 10.44 & -10.2 & 10.32 \\
无传动 & 7.77 & -8.17 & 7.97 & & 8.02 & -8.42 & 8.22 \\
\hline
\end{tabular}

\section{6 结论}

(1) 建立包含齿轮传动系统的高速动车组动力 学模型是十分必要的, 也是可行的。采用所建模型, 获得齿轮啮合刚度、齿轮径向力、轴向力以及圆周 力等载荷。

(2) 轮轨激扰和列车运行速度对齿轮载荷有明 显影响, 且随着车辆运行速度提高, 齿轮各种载荷 明显增大。

(3) 齿轮啮合振动对构架、电动机以及齿轮箱 体振动有一定的影响, 但对车体振动几乎没有影响。 即齿轮啮合振动在一定程度上加剧了电动机和齿轮 箱横向振动和电动机垂向振动, 同时由于齿轮的啮 合特性, 使得电动机和齿轮箱的高频振动有所体现。

所建高速轨道车辆-齿轮传动系统耦合动力学 模型, 对获得更为准确的高速车辆系统动力性能、 齿轮传动系统振动及载荷等具有重要意义, 后期拟 在进一步建立包含轴承系统振动模型的基础上, 更 深层次地研究高速动车组-传动系统动力特性。

\section{参 考 文 献}

[1] 张建全, 陶功安, 孔媛媛, 等. 牵引力对城轨车辆动力 学性能的影响 [J]. 机械工程与自动化, 2017(3): 22-24. 
ZHANG Jianquan, TAO Gongan, KONG Yuanyuan, et al. Effect of traction force on dynamic performance of mass transit vehicles[J]. Mechanical Engineering \& Automation, 2017(3): 22-24.

[2] 任尊松. 车辆动力学基础[M]. 北京: 中国铁道出版社, 2009.

REN Zunsong. Foundation of railway vehicle dynamics[D]. Beijing: China Railway Publishing House, 2009.

[3] 任尊松, 孙守光, 李强, 等. 构架结构振动与动态应力 仿真研究[J]. 机械工程学报, 2004, 40 (8): 187-192. REN Zunsong, SUN Shouguang, LI Qiang, et al. Simulation of railway bogie dynamic stress and elastic vibration[J]. Journal of Mechanical Engineering, 2004, 40 (8): 187-192.

[4] 王文静, 孙守光, 李强. 柔性构架的动应力仿真 $[\mathrm{J}]$. 铁 道学报, 2006, 28(1): 44-49.

WANG Wenjing, SUN Shoiuguang, LI Qiang. Dynamic stress simulation of flexible bogie frame[J]. Journal of the China Railway Society, 2006, 28(1): 44-49.

[5] REN Zunsong. Study on the multi-point of the high speed vehicle-turnout system dynamics. Chinese Journal of Mechanical Engineering, 2013, 26(3): 518-525.

[6] CHEN Zaigang, ZHAI Wanming, WANG Kaiyun. Dynamic investigation of a locomotive with effect of gear transmissions under tractive conditions[J]. Journal of Sound and Vibration, 2017, 408: 220-233.

[7] HU Weigang, LIU Zhiming, LIU Dekun, et al. Fatigue failure analysis of high speed train gearbox housings[J]. Engineering Failure Analysis, 2017, 73: 57-71.

[8] TANG Zhaoping, TANG Shuai, SUN Jianping, et al. Multi-condition contact stress analysis of high speed train helical gear[J]. Periodica Polytechnica Transportation Engineering, 2016, 44(4): 193-200.

[9] AL-SHYYABA A, KAHRAMANA. Non-linear dynamic analysis of a multi-mesh gear train using multi-term harmonic balance method: sub-harmonic motions[J]. Journal of Sound and Vibration, 2005， 279: 417-451.

[10] WANG Junguo, HE Guangyue, ZHANG Jie, et al. Nonlinear dynamics analysis of the spur gear system for railway locomotive[J]. Mechanical Systems and Signal Processing, 2017, 85: 41-55.

[11] 张睿, 张义民, 朱丽莎, 等. 齿轮传动激励下采煤机摇 臂振动特性[J]. 东北大学学报, 2018(1): 108-111, 117. ZHANG Rui, ZHANG Yimin, ZHU Lisha, et al. Vibration characteristics for the rocker arm of shearer with gear meshing excitation[J]. Journal of Northeast University, 2018(1): 108-111, 117.

[12] 杨军. 齿轮系统轮齿啮合过程的动力学分析 [J]. 机械 传动, 2011(8): 29-34.

YANG Jun. Dynamics analysis of tooth meshing process of gear system[J]. Mechanical Transmission, 2011(8): 29-34.

[13] 金思勤, 赵永强, 李枫, 等. 高速动车组齿轮箱加载试 验及故障诊断研究 $[\mathrm{J}]$. 机车车辆工艺, 2014(4): 1-3. JIN Siqin, ZHAO Yongqiang, LI Feng, et al. Research on load test and failure diagnosis of the gearbox for high speed EMU[J]. Locomotive and Rolling Stock Technology, 2014(4): 1-3.

[14] 陆正刚, 胡用生. 基于刚柔耦合系统的关键零部件动应 力仿真和疲劳寿命计算 $[J]$. 铁道车辆, 2006, 44(1): 6-11.

LU Zhenggang, HU Yongsheng. Dynamic stress simulation and fatigue life calculation of key parts $\&$ components based on rigid flexible coupling system[J]. Rolling Stock, 2006, 44(1): 6-11.

[15] 王燕, 刘建新, 李丞. 计及齿轮时变啮合刚度的机车驱 动系统振动稳定性[J]. 振动与冲击, 2017(16): 100- 105. WANG Yan, LIU Jianxin, LI Miao. Vibration stability for a locomotive driving system with time-varying mesh stiffness of gears[J]. Journal of Vibration and Shock, 2017(16): 100-105.

[16] 李添翼, 武志斐, 王铁, 等. 基于 Workbench 的齿轮啮 合振动分析[J]. 科学技术与工程, 2017(24): 49-54.

LI Tianyi, WU Zhifei, WANG Tie, et al. Analysis of gear meshing vibration based on workbench[J]. Science Technology and Engineering, 2017(24): 49-54.

[17] 程广利, 朱石坚, 黄映云, 等. 齿轮箱振动测试与分析 [J]. 海军工程大学学报, 2004(6): 83-88.

CHENG Guangli, ZHU Shijian, HUANG Yingyun, et al. Vibration measurement and analysis of gearbox[J]. Journal of Naval University of Engineering, 2004(6): $83-88$.

[18] 许思思, 黄冠华, 姜海博, 等. 高速列车齿轮箱振动烈 度评价方法探讨[J]. 铁道技术监督, 2017(11)：30-32， 49.

XU Sisi, HUANG Guanhua, JIANG Haibo, et al. Evaluate the gearbox vibration severity of high speed $\operatorname{train}[\mathrm{J}]$. Railway Quality Control, 2017(11): 30-32， 49.

[19] 安妮, 徐建民. 齿轮箱振动的故障诊断与分析[J]. 武汉 工程大学学报, 2011(12): 70-72, 88.

AN Ni, XU Jianmin. Fault diagnosis and analysis of 
vibration of gearbox[J]. Journal of Wuhan Institute Technology, 2011(12): 70-72, 88.

[20] 孙刚. 高速列车传动系统动力学研究[D]. 北京: 北京交 通大学, 2018.

SUN Gang. Study on dynamic performance of high speed vehicle transmission system[D]. Beijing: Beijing Jiaotong
University, 2018.

作者简介: 孙刚, 男, 1992 年出生。主要研究方向为高速列车齿轮传动 系统动力学。

E-mail: xingtianliu@bjtu.edu.cn

任尊松(通信作者), 男, 1969 年出生, 博士, 教授, 博士研究生导师。 主要研究方向轨道车辆动力学、结构强度及可靠性。

E-mail: zsren@bjtu.edu.cn 